\section{MICROBIAL CONVERSION OF MILBEMYCINS: 28-HYDROXYLATION OF MILBEMYCINS BY Amycolata autotrophica}

\author{
Keiko Nakagawa ${ }^{\dagger}$, Kazuo Sato ${ }^{\dagger \dagger}$, \\ YoshIHISA TSUKAMOTo ${ }^{\dagger \dagger}$ and AKIO TORIKATA ${ }^{\dagger}$ \\ † Fermentation Research Laboratories, \\ Sankyo Co., Ltd., \\ 1-2-58 Hiromachi, Shinagawa-ku, Tokyo 140, Japan \\ ${ }^{\dagger \dagger}$ Agricultural Chemicals Research Laboratories, \\ Sankyo Co., Ltd. \\ 1041 Yasu, Yasu-cho, Shiga 520-23, Japan
}

(Received for publication September 24, 1992)

Milbemycins are a family of sixteen-membered macrolides produced by Streptomyces hygroscopicus subsp. aureolacrimosus. They exhibit broad-spectrum insecticidal and acaricidal activity. ${ }^{1 \sim 3)}$

In the course of our studies on the microbial conversion of milbemycins, we obtained $13 \beta$ hydroxymilbemycins $\mathrm{A}_{4}, \mathrm{~A}_{3}, \mathrm{D}, 13 \beta$-hydroxy-LLF28249 $\alpha, 28$-hydroxymilbemycin D, and 28-hydroxy-LL-F28249 $\alpha$ by using Cunninghamella echinulata. ${ }^{4)}$ In the former effort, some of the microorganisms were estimated to convert milbemycin $\mathrm{A}_{4}$ (1a) into 28-hydroxy derivative (1b) which was anticipated as a useful compound for synthesizing new milbemycin derivatives. However, the low conversion yield prevented isolation and structuredetermination of the fermentation mixtures. We continued the screening to find a microorganism which possessed higher hydroxylation activity. Consequently Amycolata autotrophica subsp.

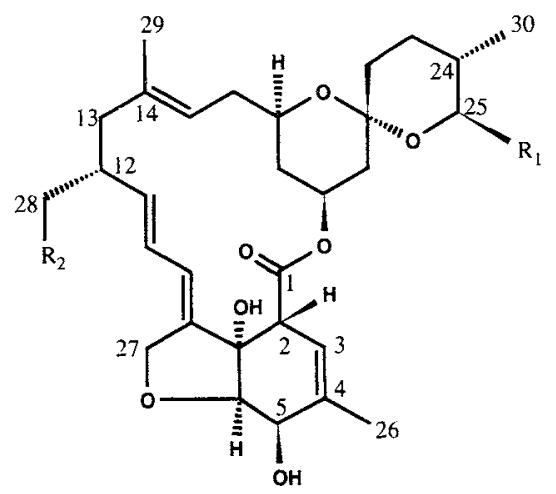

$$
\begin{array}{lll}
\text { 1a } & \mathrm{R}_{1}=\mathrm{CH}_{2} \mathrm{CH}_{3} & \mathrm{R}_{2}=\mathrm{H} \\
\text { 1b } & \mathrm{R}_{1}=\mathrm{CH}_{2} \mathrm{CH}_{3} & \mathrm{R}_{2}=\mathrm{OH} \\
\text { 2a } & \mathrm{R}_{1}=\mathrm{CH}_{3} & \mathrm{R}_{2}=\mathrm{H} \\
\text { 2b } & \mathrm{R}_{1}=\mathrm{CH}_{3} & \mathrm{R}_{2}=\mathrm{OH}
\end{array}
$$

canberrica ATCC 35203 was found to convert milbemycin $\mathrm{A}_{4}$ (1a) into 28-hydroxymilbemycin $\mathrm{A}_{4}$ (1b) efficiently.

The present paper deals with the 28-hydroxylation of milbemycin $\mathrm{A}_{4}$ (1a) and $\mathrm{A}_{3}$ (2a) by A. autotrophica ATCC 35203.

Converted milbemycins were detected by TLC (Merck Art. 5715: EtOAc) and HPLC (column: Waters, Nova pak $\mathrm{C}_{18} 8 \mathrm{~mm} \times 10 \mathrm{~cm}$; solvent: system 1 , acetonitrile - water $(75: 25)$, with a flow rate of $1.5 \mathrm{ml} /$ minute; system 2 , acetonitrile - water $(55: 45)$, with a flow rate of $1.0 \mathrm{ml} /$ minute; detector: UV $243 \mathrm{~nm}$ ).

A. autotrophica ATCC 35203 was cultured in twenty $500-\mathrm{ml}$ Erlenmyer flasks containing $100 \mathrm{ml}$ MY medium composed of $1.0 \%$ of glucose, $0.5 \%$ of Polypepton (Daigo Nutritive Chemicals), $0.3 \%$ of yeast extract (Difco), and $0.3 \%$ of malt extract (Difco) $(\mathrm{pH} 6.3 \sim 6.5)$, at $28^{\circ} \mathrm{C}$ on a rotary shaker (200 220 rpm). After 2 days cultivation, milbemycin $\mathrm{A}_{4}(5 \%[\mathrm{w} / \mathrm{v}]$ in 1,4-dioxane) was added to a final concentration of $250 \mu \mathrm{g} / \mathrm{ml}$ and cultivation was continued for seven additional days. Then the culture broth was extracted with three 1,000-ml portions of EtOAc. The EtOAc extract was dried over anhydrous sodium sulfate and evaporated. This extract was then purified by silica gel chromatography $(20 \sim 90 \%$ EtOAc in $n$-hexane as an eluent) to give $32 \mathrm{mg}(6.2 \%)$ of 28 -hydroxymilbemycin $\mathrm{A}_{4}$ (1b).

Milbemycin $\mathrm{A}_{3}$ (2a) $(500 \mathrm{mg})$ which is a congener of milbemycin $A_{4}(\mathbf{1 a})$, was subjected to the similar conversion conditions as used for milbemycin $\mathrm{A}_{4}$, and $11 \mathrm{mg}(2.1 \%)$ of the corresponding 28-hydroxy derivative was obtained. The Rf values on TLC and HPLC retention times of 28-hydroxy derivatives are listed in Table 1. The physico-chemical properties of 28-hydroxymilbemycin $A_{4}$ (1b) and $A_{3}$ (2b)

\begin{tabular}{|c|c|c|c|}
\hline \multirow{2}{*}{ Compound $^{\mathrm{a}}$} & \multirow{2}{*}{$\begin{array}{l}\text { TLC Rf }{ }^{b} \\
\text { values }\end{array}$} & \multicolumn{2}{|c|}{ HPLC Rt's (minutes) } \\
\hline & & System 1 & System 2 \\
\hline $1 \mathrm{a}$ & 0.59 & 16.07 & - \\
\hline $1 \mathrm{~b}$ & 0.18 & 4.97 & 18.26 \\
\hline $\mathbf{2 a}$ & 0.59 & 11.80 & - \\
\hline $\mathbf{2 b}$ & 0.18 & 3.98 & 12.29 \\
\hline
\end{tabular}
were as follows:

Table 1. TLC Rf values and HPLC retention times of milbemycins and conversion products. 
28-Hydroxymilbemycin $\mathrm{A}_{4}(\mathbf{1 b})$ : IR ( $\left.\mathrm{KBr}\right) \mathrm{cm}^{-1}$ $3650 \sim 3150$ (br s), 2957 (s), 2928 (s), 2873 (s), 1715 (s), $\mathrm{cm}^{-1} ;{ }^{1} \mathrm{H} \mathrm{NMR}\left(270 \mathrm{MHz}, \mathrm{CDCl}_{3}\right) \delta 5.67 \sim 5.94$ $(2 \mathrm{H}, \mathrm{m}, 9-\mathrm{H}, 10-\mathrm{H}), 5.25 \sim 5.44(3 \mathrm{H}, \mathrm{m}, 3-\mathrm{H}, 11-\mathrm{H}$, $19-\mathrm{H}), 5.01(1 \mathrm{H}, \mathrm{t}, J=7.7 \mathrm{~Hz}, 15-\mathrm{H}), 4.67$ and 4.74 $\left(2 \mathrm{H}, \mathrm{ABq}, J=14.3 \mathrm{~Hz}, 27-\mathrm{H}_{2}\right), 4.29(1 \mathrm{H}$, br s, $5-\mathrm{H})$, $4.16(1 \mathrm{H}, \mathrm{s}, 7-\mathrm{OH}), 3.96(1 \mathrm{H}, \mathrm{d}, J=6.1 \mathrm{~Hz}, 6-\mathrm{H})$, $3.51 \sim 3.72(2 \mathrm{H}, \mathrm{m}, 17-\mathrm{H}, 28-\mathrm{H}), 3.39(1 \mathrm{H}, \mathrm{dd}$, $J=8.1,10.9 \mathrm{~Hz}, 28-\mathrm{H}), 3.27(1 \mathrm{H}, \mathrm{dd}, J=2.2,4.6 \mathrm{~Hz}$, $2-\mathrm{H}), 3.08\left(1 \mathrm{H}, \mathrm{dt}, J_{\mathrm{d}}=2.8 \mathrm{~Hz}, J_{\mathrm{t}}=9.2 \mathrm{~Hz}, 25-\mathrm{H}\right)$, $2.46 \sim 2.59(1 \mathrm{H}, \mathrm{m}, 12-\mathrm{H}), 2.16 \sim 2.38(4 \mathrm{H}, \mathrm{m}, 5-\mathrm{OH}$, $\left.13-\mathrm{H}, 16-\mathrm{H}_{2}\right), 2.01(1 \mathrm{H}$, ddd, $J=1.6,5.0,12.1 \mathrm{~Hz}$, $20-\mathrm{H}), 1.87\left(3 \mathrm{H}, \mathrm{s}, 26-\mathrm{H}_{3}\right), 1.55\left(3 \mathrm{H}, \mathrm{s}, 29-\mathrm{H}_{3}\right)$, $1.22 \sim 1.94\left(10 \mathrm{H}, \mathrm{m}, 13-\mathrm{H}, 18-\mathrm{H}, 20-\mathrm{H}, 22-\mathrm{H}_{2}\right.$, $\left.23-\mathrm{H}_{2}, 24-\mathrm{H}, 31-\mathrm{H}_{2}\right), 0.99\left(3 \mathrm{H}, \mathrm{t}, J=7.3 \mathrm{~Hz}, 32-\mathrm{H}_{3}\right)$, $0.80 \sim 0.95(1 \mathrm{H}, \mathrm{m}, 18-\mathrm{H}), 0.83(3 \mathrm{H}, \mathrm{d}, J=6.5 \mathrm{~Hz}$, $\left.30-\mathrm{H}_{3}\right)$; MS $m / z 558\left(\mathrm{M}^{+}, \mathrm{C}_{32} \mathrm{H}_{46} \mathrm{O}_{8}\right), 430,412$, $372,330,288,264,245,195,167$; HREI-MS calcd for $\mathrm{C}_{32} \mathrm{H}_{46} \mathrm{O}_{8}: 558.3193$, found: 558.3183 .

28-Hydroxymilbemycin $\mathrm{A}_{3}$ (2b): IR (KBr) $\mathrm{cm}^{-1}$ $3650 \sim 3100$ (brs), 2968 (s), 2927 (s), 2873 (s), 1719 (s); ${ }^{1} \mathrm{H} \mathrm{NMR}\left(270 \mathrm{MHz}, \mathrm{CDCl}_{3}\right) \delta 5.75 \sim 5.96(2 \mathrm{H}$, m, 9-H, 10-H), 5.26 5.43 (3H, m, 3- H, 11- H, 19-H), $5.04(1 \mathrm{H}, \mathrm{t}, J=7.8 \mathrm{~Hz}, 15-\mathrm{H}), 4.67$ and $4.73(2 \mathrm{H}$, $\left.\mathrm{dABq}, J_{\mathrm{d}}=2.0 \mathrm{~Hz}, J_{\mathrm{ABq}}=15.5 \mathrm{~Hz}, 27-\mathrm{H}_{2}\right), 4.29(1 \mathrm{H}$, $\mathrm{d}, J=5.6 \mathrm{~Hz}, 5-\mathrm{H}), 4.10 \sim 4.25(1 \mathrm{H}$, brs, $7-\mathrm{OH})$, $3.95(1 \mathrm{H}, \mathrm{d}, J=5.6 \mathrm{~Hz}, 6-\mathrm{H}), 3.50 \sim 3.61(1 \mathrm{H}, \mathrm{m}$, $17-\mathrm{H}), 3.55(1 \mathrm{H}, \mathrm{dd}, J=5.4,10.4 \mathrm{~Hz}, 28-\mathrm{H}), 3.39$ $(1 \mathrm{H}, \mathrm{dd}, J=8.2,10.4 \mathrm{~Hz}, 28-\mathrm{H}), 3.21 \sim 3.30(2 \mathrm{H}, \mathrm{m}$, $2-\mathrm{H}, 25-\mathrm{H}), 2.42 \sim 2.61(1 \mathrm{H}, \mathrm{m}, 12-\mathrm{H}), 2.16 \sim 2.28$ $\left(3 \mathrm{H}, \mathrm{m}, 13-\mathrm{H}, 16-\mathrm{H}_{2}\right), 2.02(1 \mathrm{H}, \mathrm{dd}, J=4.2,11.8 \mathrm{~Hz}$, $20-\mathrm{H}), 1.87\left(3 \mathrm{H}, \mathrm{s}, 26-\mathrm{H}_{3}\right), 1.55\left(3 \mathrm{H}, \mathrm{s}, 29-\mathrm{H}_{3}\right)$, $1.20 \sim 1.92\left(8 \mathrm{H}, \mathrm{m}, 13-\mathrm{H}, 18-\mathrm{H}, 20-\mathrm{H}, 22-\mathrm{H}_{2}, 23-\mathrm{H}_{2}\right.$, $24-\mathrm{H}), 1.15\left(3 \mathrm{H}, \mathrm{d}, J=6.0 \mathrm{~Hz}, 31-\mathrm{H}_{3}\right), 0.85 \sim 1.02$ $(1 \mathrm{H}, \mathrm{m}, 18-\mathrm{H}), 0.83\left(3 \mathrm{H}, \mathrm{d}, J=6.4 \mathrm{~Hz}, 30-\mathrm{H}_{3}\right)$; MS $m / z 544\left(\mathrm{M}^{+}, \mathrm{C}_{31} \mathrm{H}_{44} \mathrm{O}_{8}\right) ; 416,398,372,330,288$, 264, 250, 231, 181, 167, 153; HREI-MS calcd for $\mathrm{C}_{31} \mathrm{H}_{44} \mathrm{O}_{8}: 544.3036$, found: 544.3049 .

We have reported that $\mathrm{C}-30, \mathrm{C}-26$, and $\mathrm{C}-29$ methyl groups of milbemycin $\mathrm{A}_{4}$ (1a) were hydroxylated by microorganisms. ${ }^{5 \sim 7)}$ In this report the 28-hydroxylation of milbemycin $\mathrm{A}_{4}$ (1a) was confirmed. Thus, all the methyl groups of milbemycin $\mathrm{A}_{4}$ can be selectively hydroxylated by microbial conversion. Further studies on microbial conversion of milbemycin $\mathrm{A}_{4}$ (1a) involving the side chain at $\mathrm{C}-25$ are in progress.

\section{References}

1) Takiguchi, Y.; H. Mishima, M. Okuda, M. Terao, A. AOKI \& R. FUKuda: Milbemycins, a new family of macrolide antibiotics: Fermentation, isolation and physico-chemical properties. J. Antibiotics 33: 1120 1127,1980

2) Okazaki, T.; M. Ono, A. Aoki \& R. Fukuda: Milbemycins, a new family of macrolide antibiotics: Producing organisms and its mutants. J. Antibiotics 36: 438 441, 1983

3) Mishima, H.; J. Ide, S. Muramatsu \& M. Ono: Milbemycins, a new family of macrolide antibiotics. Structure determination of milbemycins D, E, F, G, H, J and K. J. Antibiotics 36: 980 990, 1983

4) Nakagawa, K.; S. Miyakoshi, A. Torikata, K. SATO \& Y. TSUKamoTo: Microbial conversion of milbemycins: Hydroxylation of milbemycin $\mathrm{A}_{4}$ and related compounds by Cunninghamella echinulata ATCC 9244. J. Antibiotics 44: 232 240, 1991

5) Nakagawa, K.; A. Torikata, K. Sato \& Y. TSUKAMOTO: Microbial conversion of milbemycins: 30-Oxidation of milbemycin $\mathrm{A}_{4}$ and related compounds by Amycolata autotrophica and Amycolatopsis mediterranei. J. Antibiotics 43: $1321 \sim 1328$, 1990

6) Nakagawa, K; K. Sato \& T. Okazaki \& A. TORIKATA: Microbial conversion of milbemycins: $13 \beta, 29$-Dihydroxylation of milbemycins by soil isolate Streptomyces cavourensis. J. Antibiotics 44: $803 \sim 805,1991$

7) Nakagawa, K.; K. Sato, Y. Tsukamoto \& A. TORIKATA: Microbial conversion of milbemycins: 29-Hydroxylation of milbemycins by genus Syncephalastrum. J. Antibiotics 45: 802 805, 1992 